\title{
The impact of left ventricular geometry on left atrium phasic function in obstructive sleep apnea syndrome: a multimodal echocardiography investigation
}

\author{
Yong Zhang ${ }^{1}$, Wen Shui ${ }^{1}$, Yun Tian' ${ }^{1}$, Zhenxia Zhang ${ }^{2}$, Juan Li ${ }^{1}$ and Jian Wang ${ }^{1 *}$
}

\begin{abstract}
Background: Left ventricular geometry and left atrium (LA) enlargement are risk factors for cardiovascular disease. However, reports on the relationship between left ventricular geometry and LA volume yielded contradictory findings, and LA phasic function remains unclear. Hence, this study aimed to investigate the influence of left ventricular geometry on LA volume and phasic function in patients with obstructive sleep apnea syndrome (OSAS) via a multimodal echocardiographic approach.
\end{abstract}

Methods: In this cross-sectional study, 221 patients with OSAS (age 20-68 years, mean age 45.27 12.50 years) underwent clinical evaluation, polysomnography, and multimodal echocardiographic examination with two-dimensional echocardiography (2DE), two-dimensional speckle-tracking echocardiography (2D-STE) and three-dimensional echocardiography (3DE). Based on conventional classification of left ventricular geometry, patients with OSAS were divided into four groups: normal geometry (NG), concentric remodeling (CR), concentric hypertrophy $(\mathrm{CH})$, and eccentric hypertrophy (EH).

Results: Based on 2DE and 3DE, the LA volumes and indices gradually increased from NG to CH. Additionally, 2DE and 3DE LA maximum volume index (LAVImax) were higher in patients with $\mathrm{CH}$ and $\mathrm{EH}$ than in patients with NG and $C R(P<0.05)$. The reservoir function, estimated by $L A$ total emptying fraction ( $L A$ TotEF) was lower in patients with $\mathrm{CH}$ than in patients with NG in 2DE and 3DE (both, $P<0.05$ ). Also, LA conduit function, evaluated by LA passive emptying fraction (LA PassEF) was lower in patients with $\mathrm{CH}$ than in patients with $\mathrm{NG}$ and $\mathrm{CR}$, and in patients with $\mathrm{EH}$ than in those with NG in 2DE and 3DE (all, $P<0.05$ ). The LA booster pump function, evaluated by LA active emptying fraction (LA ActEF) showed no statistically significant difference in $2 \mathrm{DE}$; however, it was greater in patients with $\mathrm{CH}$ than in those with NG in 3DE. Similar results were obtained by 2D-STE, and CH was significantly associated with LA strain during systole (LAS-S, $\beta=-0.546,95 \% \mathrm{Cl}:-6.371-(-3.444)$; $P<0.001)$, early diastole (LAS-E, $\beta=-0.636,95 \% \mathrm{Cl}$ : $-9.532-$ $(-5.710) ; P<0.001)$, and late diastole (LAS-A, $\beta=-0.450,95 \% \mathrm{Cl}: 1.518-3.909 ; P<0.001)$ in multiple linear regression.

Conclusions: The LA phasic function changed with left ventricular geometry via multimodal echocardiography. CH had the most notable negative effect on the maximum volume and phasic function of the LA.

\footnotetext{
*Correspondence: wangjian5688@126.com

${ }^{1}$ Medical imaging department of Shanxi Medical University; Department

of Ultrasound, First Hospital of Shanxi Medical University, 85 Jiefang Nan

Road, Taiyuan 030001, Shanxi, China

Full list of author information is available at the end of the article
}

(C) The Author(s) 2021. Open Access This article is licensed under a Creative Commons Attribution 4.0 International License, which permits use, sharing, adaptation, distribution and reproduction in any medium or format, as long as you give appropriate credit to the original author(s) and the source, provide a link to the Creative Commons licence, and indicate if changes were made. The images or other third party material in this article are included in the article's Creative Commons licence, unless indicated otherwise in a credit line to the material. If material is not included in the article's Creative Commons licence and your intended use is not permitted by statutory regulation or exceeds the permitted use, you will need to obtain permission directly from the copyright holder. To view a copy of this licence, visit http://creativecommons.org/licenses/by/4.0/. The Creative Commons Public Domain Dedication waiver (http://creativeco mmons.org/publicdomain/zero/1.0/) applies to the data made available in this article, unless otherwise stated in a credit line to the data. 
Keywords: Obstructive sleep apnea, Left ventricular geometry, Left atrium, Phasic function, Speckle-tracking echocardiography, Three-dimensional echocardiography

\section{Background}

Obstructive sleep apnea syndrome (OSAS) is a prevalent sleep-related breathing disorder and an independent risk factor for cardiovascular disease [1]. Notably, abnormal left ventricular geometry with diastolic dysfunction and left atrium (LA) enlargement are common symptoms among patients with OSAS [2-4]. These have also been identified in patients without underlying cardiovascular disease [5]. Moreover, both conditions contribute to cardiovascular morbidity and mortality [6].

Multiple previous studies have revealed that left ventricular geometry is independently associated with LA enlargement. A study using the linear method of two-dimensional echocardiography reported that LA enlargement was related to the presence of eccentric hypertrophy [7]. Another study reported that LA enlargement, evaluated by LA volume through the arealength method of two-dimensional echocardiography, was significantly associated with concentric hypertrophy compared to eccentric hypertrophy [8]. Similarly, a study using the area-length method of two-dimensional echocardiography with larger sample sizes revealed that left atrium enlargement was associated with concentric hypertrophy and eccentric hypertrophy [9]. Furthermore, a previous study reported that LA enlargement estimated by diameter or volume was closely related to left ventricular mass index but was not related to left ventricular geometry [10].

Nevertheless, previous studies evaluated LA function using a single echocardiographic method and yielded contradictory findings. Additionally, existing echocardiographic techniques, including two-dimensional speckle tracking technique and three-dimensional echocardiography, are useful for evaluating LA size and phase function [11]. Therefore, our study was designed to investigate the influence of left ventricular geometry on LA enlargement and LA phasic function combined with various echocardiographic methods in patients with OSAS.

\section{Methods}

\section{Study population}

This cross-sectional study was approved by the Institutional Ethics Committee of Shanxi Medical University First Hospital. All patients were enrolled after providing written informed consent and all underwent physical examination, overnight polysomnography, blood biochemistry, and multimodal echocardiography.
Between January 2019 and December 2019, a total of 221 patients diagnosed with OSAS from Sleep Units in the First Hospital of Shanxi Medical University were enrolled (167 men, 54 women; age, 20-68 years) (Fig. 1).

The inclusion criteria were age $>18$ years and patients with an apnea-hypopnea index $(\mathrm{AHI}) \geq 5$ events/hour. Briefly, AHI is the sum of the average number of apnea and hypopnea per hour [12]. Apnea is defined as a loss or significant decrease ( $\geq 90 \%$ from baseline) of oral and nasal airflow during sleep for $\geq 10 \mathrm{~s}$. Hypopnea is defined as a $30 \%$ or more increase in oral-nasal airflow from baseline during sleep accompanied by a $3 \%$ or more decrease in blood oxygen saturation for a duration $\geq 10 \mathrm{~s}$. The exclusion criteria included the following: myocardial infarction, heart failure, arrhythmia, coronary heart disease (CHD), moderate or severe valvular regurgitation or stenosis, left ventricular ejection fraction $<50 \%$ (modified Simpson method), moderate or severe hepatic and renal disease, chronic obstructive pulmonary disease (COPD), hypothyroidism or hyperthyroidism, and neoplastic disease. Moreover, patients who received continuous positive airway pressure (CPAP) or surgical treatment and had unsatisfactory echocardiographic images were excluded from the study.

Physical examination was performed in all patients. Blood pressure (systolic blood pressure [SBP] and diastolic blood pressure [DBP]) was measured using a mercury sphygmomanometer on the naked right arm with patients in a seated point position after 30 min of rest. Hypertension was defined as systolic blood pressure $(\mathrm{BP}) \geq 140 \mathrm{mmHg}$ or diastolic blood pressure $\geq 90 \mathrm{mmHg}$ or being treated with hypertensive medication [13]. According to the $1999 \mathrm{WHO}$ diagnostic criteria, diabetes was defined as FPG $\geq 7.0 \mathrm{mmol} / \mathrm{L}$ and/or blood glucose $\geq 11.1 \mathrm{mmol} / \mathrm{L}$ at $2 \mathrm{~h}$ postprandial or having been treated with diabetes medication. Obesity was defined as body mass index $(\mathrm{BMI}) \geq 28 \mathrm{~kg} / \mathrm{m}^{2}$, and dyslipidemia was defined as total cholesterol (TC) $\geq 5.17 \mathrm{mmol} / \mathrm{L}$, triglycerides (TG) $\geq 1.7 \mathrm{mmol} / \mathrm{L}$, high-density lipoprotein $(\mathrm{HDL})<1.03 \mathrm{mmol} / \mathrm{L}$, and low-density lipoprotein $(\mathrm{LDL}) \geq 3.33 \mathrm{mmol} / \mathrm{L}[14]$. Body mass index (BMI) was calculated as the weight divided by height squared.

Blood samples were drawn from the peripheral vein the morning after PSG. Total cholesterol (TC), triglycerides (TG), high-density lipoprotein (HDL), low-density lipoprotein (LDL), and fasting blood glucose (Glu) levels were measured using an automatic biochemical analyzer (OLYMPUSAU5400, Japan). 


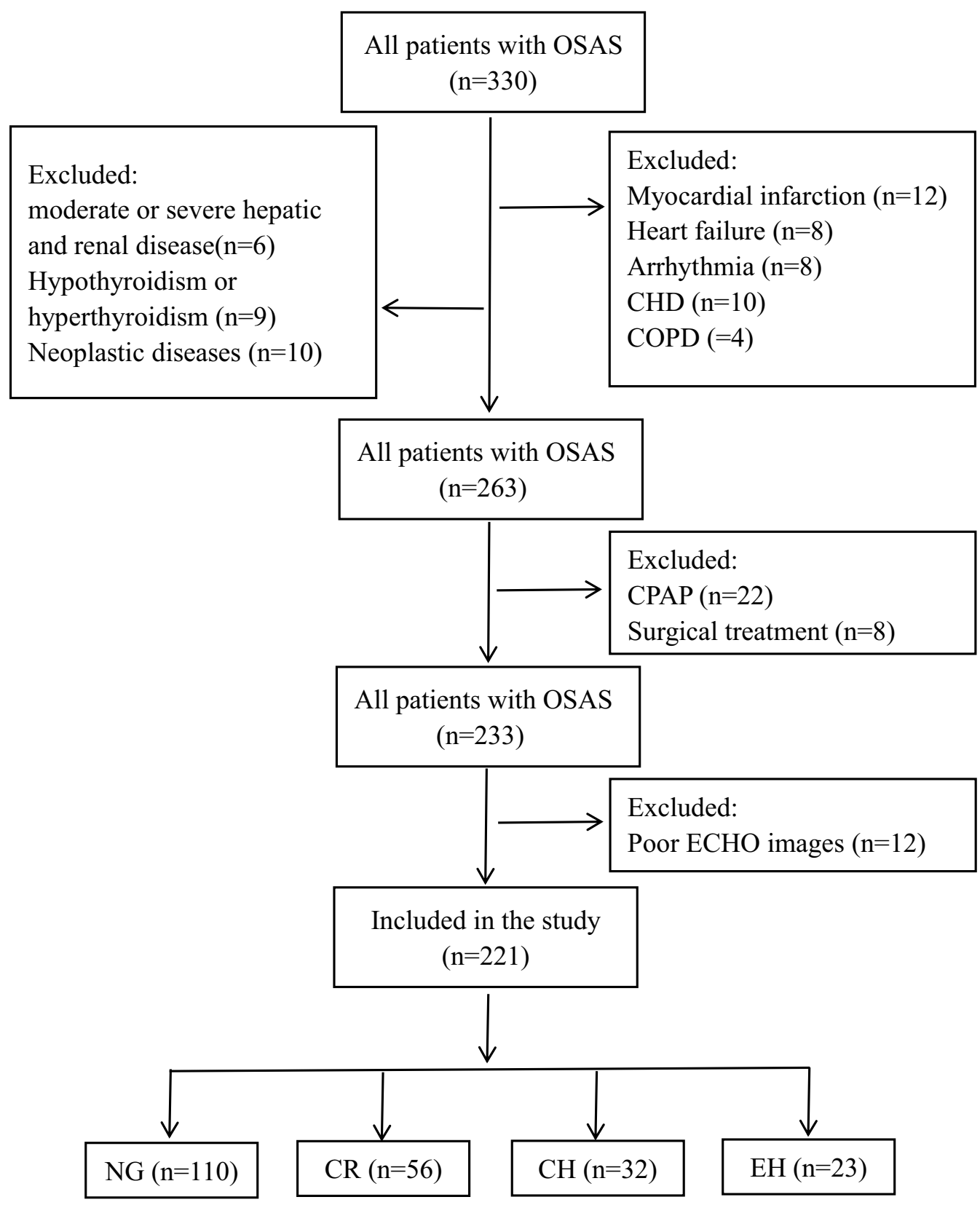

Fig. 1 Study flowchart. OSAS: Obstructive sleep apnea syndrome; COPD: Chronic obstructive pulmonary disease; CPAP: continuous positive airway pressure; CHD: Coronary heart disease; ECHO: Echocardiography

\section{Polysomnography}

All patients underwent overnight polysomnography in sleep units using standard recording techniques (SOMNO screen V series; German). Sleep recordings, including the apnea-hypopnea index (AHI), mean oxygen saturation (Mean- $\mathrm{SaO}_{2}$ ), lowest oxygen saturation (lowest $\mathrm{SaO}_{2}$ ), and percentage of total sleep time when blood oxygen saturation was less than 90\% (T90), were obtained by a skilled technician based on the American
Academic Sleep Medicine (AASM) 2017 criteria [15]. The AHI was defined as the number of apnea and hypopnea events per hour of sleep, and more than five were required for diagnosis.

\section{Echocardiography}

Based on the recommendations of the American Society of Echocardiography [16], all patients underwent transthoracic echocardiographic examinations, including 
two-dimensional echocardiography (2DE), two-dimensional speckle-tracking echocardiography (2D-STE), and three-dimensional echocardiography (3DE), using EPIQ 7C Color Doppler Ultrasound (PHILIPS), with a S5-1 and X5-1 probe and a frequency of 1.0-5.0 MHz. All patients were positioned in the left lateral decubitus position and connected to an electrocardiogram (ECG).

Left ventricular end-diastolic diameter (LVEDD), interventricular septum thickness (IVST), and left ventricular posterior wall thickness (LVPWT) were measured on the parasternal long-axis view. The early diastolic peak flow velocity $(\mathrm{E})$ and late diastolic peak flow velocity (A) were measured using conventional pulsed Doppler imaging in the apical four-chamber view. Tissue Doppler imaging on the apical four-chamber view was used to measure mitral annular early diastolic myocardial velocity (e). Left ventricular ejection fraction was calculated using Simpson's biplane method; meanwhile, left ventricular mass (LVM) was calculated while the left ventricular mass index (LVMI) was indexed to the body surface area by using the following formula [16]: $0.8 \times 1.04$ [ $(\text { LVEDD + IVST + PWT })^{3}$-LVEDD $\left.\left.{ }^{3}\right]\right)+0.6$. The cutoff values of LVMI were $95 \mathrm{~g} / \mathrm{m}^{2}$ for women and $115 \mathrm{~g} /$ $\mathrm{m}^{2}$ for men. Relative wall thickness (RWT), with a cutoff value of 0.42 [13], was calculated using the following formula: $2 \times$ LVPWT/LVEDD.

Left ventricular geometric patterns were defined based on LVMI and RWT [16]: normal geometry (NG, normal LVMI and RWT), concentric remodeling (CR, normal LVMI, and increased RWT), concentric hypertrophy ( $\mathrm{CH}$, increased LVMI and RWT), and eccentric hypertrophy (EH, increased LVMI, and normal RWT).

\section{Two-dimension echocardiography (2DE) assessment of the left atrium volumes}

The LA volumes (LAVs), including left atrial maximum volume (LAVmax), left atrium minimum volume (LAVmin), and left atrial pre-contraction volume (LAVpre), were measured using a biplane method in four-chamber and two-chamber views. The LAVmax was measured just before the mitral valve opening (the terminal of the T wave on the ECG), LAVmin was measured just after mitral valve closure (the peak of the $R$ wave on the ECG), and LAVpre was measured at the onset of atrial systole (the peak of the P wave on the ECG). All LA volumes were indexed to body surface area (BSA).

\section{Two-dimensional speckle-tracking echocardiography (2D-STE) assessment of the left atrium strain}

All dynamic images of the four-chamber and two-chamber views were obtained using conventional two-dimensional echocardiography for three consecutive cardiac cycles. All images were digitally stored, while offline images were analyzed using QLAB-Philips software (version 10; Philips Medical Systems).

The software automatically tracked the LA endocardium and manually adjusted the region of interest when speckle tracking was insufficient. The software then calculated six segments of the LA strain and its overall strain. Finally, the overall LA strain, including LA strain during systole (LAS-S) and LA strain during late diastole (LAS-A), combined the average of the values obtained for the four-chamber and two-chamber views. The LAS-S was measured when the aortic valve was closed, while LAS-A was measured at the beginning of the $\mathrm{P}$ wave of the ECG. The LA strain during early diastole (LAS-E) was defined as the difference between LAS-S and LAS-A [17].

\section{Three-dimensional echocardiography (3DE) assessment of the left atrium volumes}

An X5-1 matrix-array transducer was used to acquire the "full-volume" for 4 consecutive cardiac cycles, and all images were digitally stored; meanwhile, they were analyzed offline using the QLAB-Philips software (version 10; Philips Medical Systems). The LA volumes were analyzed using the following anatomical landmarks: the septal, lateral, anterior, and posterior points on the atrial surface of the mitral annulus and the higher point of the LA roof. The LA appendages and pulmonary veins were excluded. Subsequently, the software automatically tracked the LA endocardium and manually adjusted the region of interest when necessary. Finally, the volumetime curve of the LA was obtained [18]. Measurement of LAVs was similar to measurement of the two-dimensional echocardiography as described above.

Based on the above LAVs, the parameters of LA function were calculated as follows [19]: LA total emptying volume (LA TotEV): LAVmax- LAVmax; LA passive emptying volume (LA PassEV): LAVmax- LAVpre; LA active emptying volume (LA ActEV): LAVpre-LAVmin; LA total emptying fraction (LA TotEF): LA TotEV/LAV$\max \times 100$; LA passive emptying fraction (LA PassEF): LA PassEV/LAVmax $\times 100$; LA active emptying fraction (LA ActEF): LA ActEV/LAVpre $\times 100$.

\section{Statistical analysis}

Continuous variables were evaluated for normal distribution using the one-way Kolmogorov-Smirnov test. Normally distributed continuous variables were presented as mean \pm standard deviation and analyzed using one-way ANOVA with Bonferroni post hoc test. Categorical variables were presented as percentages and were analyzed using the chi-square test. Multiple linear regression analysis was used to establish the relationship between left ventricular geometry and 
LAVImax, as well as left ventricular phasic function after adjusting for age, gender, BMI, SBP, diabetes mellitus, dyslipidemia, AHI, and LAMI. A $P$-value $<0.05$ $(P<0.05)$ was considered statistically significant. All data were analyzed using SPSS 22.0 for Windows (IBM, Armonk, NY, USA). The figures were generated using GraphPad Prism 8.

\section{Results}

\section{Clinical, polysomnographic, and blood biochemical} parameters in the four left ventricular geometric patterns As shown in Table 1, we recruited 221 patients with OSAS, 110 patients with normal geometry, 56 patients with concentric remodeling, 32 patients with concentric hypertrophy, and 23 patients with eccentric hypertrophy. In contrast with normal geometry and concentric remodeling, patients with concentric hypertrophy and eccentric hypertrophy were associated with older age $(P<0.05)$. Patients with concentric hypertrophy and eccentric hypertrophy had higher values of SBP, DBP, hypertension, AHI, and T90, but had lower values of mean- $\mathrm{SaO}_{2}$ than those with normal geometry $(P<0.05)$. Regarding sex, BMI, obesity, diabetes mellitus, dyslipidemia, medications (including ARBs/ACEIs, Beta-blockers, CCB, diuretics, statin and antidiabetic), heart rate, lowest- $\mathrm{SaO}_{2}$, and blood biochemical values, no statistical difference was found among the four groups (Table 1).

\section{Echocardiographic characteristics of the left ventricle} in the four left ventricular geometric patterns

As shown in Table 2, patients with concentric hypertrophy and eccentric hypertrophy demonstrated a significantly larger LA dimension than those with normal geometry and concentric remodeling $(P<0.05)$. Similarly, both had greater interventricular septal thickness, left ventricular posterior wall thickness, and left ventricular mass index $(P<0.05)$. Furthermore, patients with concentric remodeling and concentric hypertrophy exhibited higher relative wall thickness than those with normal geometry and eccentric hypertrophy $(P<0.05)$. The E/A ratio gradually decreased, while the $\mathrm{E} / \mathrm{e}$ ratio gradually increased from normal geometry to concentric hypertrophy. Regarding LVEF, no statistical difference was found among the four groups (Table 2).

\section{Left atrium volumes and strains in the four left ventricular geometric patterns}

As demonstrated in Table 3, 2DE- and 3DE LA volumes and indices (maximum, minimum, pre-contraction) gradually increased from normal geometry to concentric hypertrophy. The 3DE-LAVmax showed a statistically significant difference among the four groups, while $2 \mathrm{DE}-\mathrm{LAVmax}$ was statistically significant among all four groups, except for concentric hypertrophy and eccentric hypertrophy. In addition, 2DE- and 3DELAVImax revealed a statistically significant difference among the other left ventricular geometries, except for normal geometry and concentric remodeling (Figs. 2

Table 1 Clinical, polysomnographic and blood biochemical parameters in the four left ventricular geometric patterns

\begin{tabular}{|c|c|c|c|c|c|}
\hline & $N G(n=110)$ & $C R(n=56)$ & $\mathrm{CH}(\mathrm{n}=32)$ & $\mathrm{EH}(\mathrm{n}=23)$ & $P$ \\
\hline Age (years) & $41.80 \pm 1.22$ & $44.27 \pm 1.45$ & $51.88 \pm 1.73^{\mathrm{ab}}$ & $55.09 \pm 2.03^{a b}$ & $<0.001$ \\
\hline Female (\%) & 24.5 & 23.2 & 25 & 26.1 & 0.994 \\
\hline $\mathrm{BMI}\left(\mathrm{kg} / \mathrm{m}^{2}\right)$ & $27.75 \pm 0.39$ & $28.38 \pm 0.53$ & $28.08 \pm 0.26$ & $28.37 \pm 0.30$ & 0.703 \\
\hline $\mathrm{SBP}(\mathrm{mmHg})$ & $129.36 \pm 1.32$ & $132.43 \pm 1.76$ & $138.50 \pm 1.73^{a}$ & $138.17 \pm 2.37^{a}$ & 0.001 \\
\hline $\mathrm{DBP}(\mathrm{mmHg})$ & $77.53 \pm 1.10$ & $79.30 \pm 1.44$ & $86.44 \pm 1.87^{\mathrm{a}}$ & $84.30 \pm 1.69^{a}$ & $<0.001$ \\
\hline HR (bpm) & $64.91 \pm 0.59$ & $66.82 \pm 0.93$ & $65.37 \pm 1.29$ & $65.87 \pm 1.66$ & 0.389 \\
\hline AHI (events/h) & $36.86 \pm 2.49$ & $46.15 \pm 3.60$ & $54.18 \pm 5.31^{\mathrm{a}}$ & $51.83 \pm 4.86^{\mathrm{a}}$ & 0.003 \\
\hline Mean-SaO ${ }_{2}(\%)$ & $92.54 \pm 2.88$ & $91.41 \pm 3.52$ & $90.89 \pm 3.78^{a}$ & $90.96 \pm 2.93^{\mathrm{a}}$ & 0.014 \\
\hline Lowest-SaO 2 (\%) & $74.95 \pm 1.33$ & $71.73 \pm 2.06$ & $69.69 \pm 2.67$ & $71.52 \pm 3.58$ & 0.251 \\
\hline T90 (\%) & $13.51 \pm 1.36$ & $19.17 \pm 2.97$ & $27.48 \pm 3.43^{a}$ & $24.05 \pm 4.34^{\mathrm{a}}$ & $<0.001$ \\
\hline $\mathrm{TC}(\mathrm{mmol} / \mathrm{L})$ & $4.54 \pm 0.06$ & $4.44 \pm 0.09$ & $4.65 \pm 0.17$ & $4.63 \pm 0.15$ & 0.561 \\
\hline TG (mmol/L) & $2.01 \pm 0.09$ & $1.92 \pm 0.10$ & $2.15 \pm 0.13$ & $1.95 \pm 0.15$ & 0.673 \\
\hline $\mathrm{HDL}$ (mmol/L) & $1.17 \pm 0.03$ & $1.13 \pm 0.04$ & $1.16 \pm 0.03$ & $1.09 \pm 0.04$ & 0.493 \\
\hline LDL (mmol/L) & $2.60 \pm 0.07$ & $2.32 \pm 0.11$ & $2.61 \pm 0.16$ & $2.72 \pm 0.22$ & 0.111 \\
\hline Glu (mmol/L) & $5.26 \pm 0.07$ & $5.24 \pm 0.14$ & $5.56 \pm 0.19$ & $5.38 \pm 0.22$ & 0.401 \\
\hline
\end{tabular}

NG: normal geometry; CR: concentric remodeling; $\mathrm{CH}$ : concentric hypertrophy; EH: eccentric hypertrophy; BMI: body mass index; SBP: systolic blood pressure; DBP: diastolic blood pressure; AHI: apnea hypopnea index; Mean-SaO2: mean oxygen saturation; Lowest-SaO2: lowest oxygen saturation; T90: percentage of total sleep time when blood oxygen saturation is less than 90\%; TC: Total cholesterol; TC: triglyceride; HDL: High-density lipoprotein; LDL: Low-density lipoprotein; Glu: Glucose ${ }^{\mathrm{a}} p<0.05$ for normal geometry; ${ }^{\mathrm{b}} p<0.05$ for concentric remodeling 
Table 2 Echocardiographic Characteristics of left ventricular in the four left ventricular geometric patterns

\begin{tabular}{|c|c|c|c|c|c|}
\hline & NG $(n=110)$ & $C R(n=56)$ & $\mathrm{CH}(\mathrm{n}=32)$ & $E H(n=23)$ & $P$ \\
\hline LAD (cm) & $3.25 \pm 0.33$ & $3.40 \pm 0.31^{\mathrm{a}}$ & $3.74 \pm 0.14^{\mathrm{ab}}$ & $3.79 \pm 0.14^{\mathrm{ab}}$ & $<0.001$ \\
\hline LVEDD (cm) & $4.72 \pm 0.03$ & $4.61 \pm 0.05$ & $4.99 \pm 0.08^{a b}$ & $5.32 \pm 0.07^{\mathrm{abc}}$ & $<0.001$ \\
\hline IVST (cm) & $0.92 \pm 0.08$ & $0.98 \pm 0.14^{\mathrm{a}}$ & $1.16 \pm 0.08^{\mathrm{ab}}$ & $1.09 \pm 0.12^{\mathrm{abc}}$ & $<0.001$ \\
\hline LVPWT (cm) & $0.87 \pm 0.08$ & $1.00 \pm 0.12^{\mathrm{a}}$ & $1.13 \pm 0.09^{\mathrm{ab}}$ & $1.02 \pm 0.17^{\mathrm{ac}}$ & $<0.001$ \\
\hline LVMI $\left(\mathrm{g} / \mathrm{m}^{2}\right)$ & $75.44 \pm 1.25$ & $82.85 \pm 2.12^{a}$ & $117.73 \pm 2.13^{\mathrm{ab}}$ & $115.69 \pm 2.14^{\mathrm{ab}}$ & $<0.001$ \\
\hline RWT & $0.37 \pm 0.02$ & $0.44 \pm 0.03^{\mathrm{a}}$ & $0.45 \pm 0.07^{\mathrm{ab}}$ & $0.39 \pm 0.05^{a b c}$ & $<0.001$ \\
\hline$E / A$ & $0.97 \pm 0.03$ & $0.93 \pm 0.04$ & $0.79 \pm 0.03^{a b}$ & $0.87 \pm 0.07^{a c}$ & 0.018 \\
\hline E/e & $6.54 \pm 0.25$ & $7.72 \pm 0.29^{\mathrm{a}}$ & $9.77 \pm 0.41^{\mathrm{ab}}$ & $8.87 \pm 0.68^{a}$ & $<0.001$ \\
\hline LVEF (\%) & $65.99 \pm 0.52$ & $66.31 \pm 0.90$ & $66.34 \pm 0.93$ & $63.71 \pm 1.73$ & 0.343 \\
\hline
\end{tabular}

LAD: left atrium dimension; LVEDD: left ventricular end-diastolic diameter; IVST: inter-ventricular septum thickness; LVPWT: left ventricular posterior wall thickness; LVMI: left ventricular mass index; RWT: relative wall thickness; LVEF: left ventricular ejection fraction

${ }^{a} p<0.05$ for normal geometry; ${ }^{b} p<0.05$ for concentric remodeling; ${ }^{c} p<0.05$ for concentric hypertrophy

Table 3 Left atrium volumes and strains in the four left ventricular geometric patterns

\begin{tabular}{|c|c|c|c|c|c|}
\hline & NG $(n=110)$ & $C R(n=56)$ & $\mathrm{CH}(\mathrm{n}=32)$ & $\mathrm{EH}(n=23)$ & $P$ \\
\hline \multicolumn{6}{|l|}{ 2DE LAV variables } \\
\hline LAVmax (ml) & $39.14 \pm 5.40$ & $41.81 \pm 8.04^{\mathrm{a}}$ & $49.26 \pm 4.47^{a b}$ & $45.90 \pm 5.56^{\mathrm{ab}}$ & $<0.001$ \\
\hline LAVImax $\left(\mathrm{ml} / \mathrm{m}^{2}\right)$ & $20.66 \pm 3.05$ & $21.88 \pm 4.45$ & $27.66 \pm 3.29^{\mathrm{ab}}$ & $24.42 \pm 4.01^{\mathrm{abc}}$ & $<0.001$ \\
\hline LAVmin (ml) & $13.25 \pm 2.40$ & $14.90 \pm 3.43^{\mathrm{a}}$ & $19.04 \pm 5.13^{a b}$ & $16.95 \pm 3.40^{\mathrm{a}}$ & $<0.001$ \\
\hline LAVImin $\left(\mathrm{ml} / \mathrm{m}^{2}\right)$ & $6.99 \pm 1.27$ & $7.80 \pm 1.90$ & $10.71 \pm 3.12^{\mathrm{ab}}$ & $9.04 \pm 2.17^{\mathrm{ac}}$ & $<0.001$ \\
\hline LAVpre (ml) & $21.36 \pm 4.03$ & $23.72 \pm 5.74^{a}$ & $31.75 \pm 4.02^{\mathrm{ab}}$ & $28.51 \pm 4.04^{\mathrm{ab}}$ & $<0.001$ \\
\hline LAVIpre $\left(\mathrm{ml} / \mathrm{m}^{2}\right)$ & $11.25 \pm 2.04$ & $12.40 \pm 3.01^{\mathrm{a}}$ & $17.82 \pm 2.59^{\mathrm{ab}}$ & $15.20 \pm 2.94^{\mathrm{abc}}$ & $<0.001$ \\
\hline LA TotEV (ml) & $25.90 \pm 3.86$ & $26.91 \pm 5.42$ & $30.22 \pm 4.63^{\mathrm{ab}}$ & $28.95 \pm 3.10^{\mathrm{a}}$ & 0.005 \\
\hline LA PassEV (ml) & $17.79 \pm 2.60$ & $18.09 \pm 3.12$ & $17.51 \pm 1.92$ & $17.39 \pm 2.37$ & 0.655 \\
\hline LA ActEV (ml) & $8.11 \pm 2.61$ & $8.82 \pm 3.30$ & $12.71 \pm 3.88^{\mathrm{ab}}$ & $11.56 \pm 1.83^{a b}$ & $<0.001$ \\
\hline \multicolumn{6}{|l|}{ 3DE LAV variables } \\
\hline LAVmax (ml) & $46.85 \pm 4.80$ & $49.00 \pm 5.47^{a}$ & $60.60 \pm 4.66^{\mathrm{ab}}$ & $53.77 \pm 3.78^{\mathrm{abc}}$ & $<0.001$ \\
\hline LAVImax $\left(\mathrm{ml} / \mathrm{m}^{2}\right)$ & $24.86 \pm 3.85$ & $25.84 \pm 4.68$ & $34.05 \pm 3.98^{\mathrm{ab}}$ & $28.60 \pm 3.59^{\mathrm{abc}}$ & $<0.001$ \\
\hline LAVmin (ml) & $18.04 \pm 3.83$ & $18.89 \pm 2.95$ & $25.57 \pm 3.31^{\mathrm{ab}}$ & $21.95 \pm 2.74^{\mathrm{abc}}$ & $<0.001$ \\
\hline LAVImin $\left(\mathrm{ml} / \mathrm{m}^{2}\right)$ & $9.60 \pm 2.43$ & $9.96 \pm 2.13$ & $14.37 \pm 2.23^{\mathrm{ab}}$ & $11.69 \pm 1.96^{\mathrm{abc}}$ & $<0.001$ \\
\hline LAVpre (ml) & $27.78 \pm 3.71$ & $30.11 \pm 5.51^{\mathrm{a}}$ & $44.36 \pm 5.76^{a b}$ & $35.25 \pm 5.54^{\mathrm{abc}}$ & $<0.001$ \\
\hline LAVIpre $\left(\mathrm{ml} / \mathrm{m}^{2}\right)$ & $14.72 \pm 2.49$ & $15.87 \pm 3.66$ & $24.93 \pm 3.91^{\mathrm{ab}}$ & $18.78 \pm 3.68^{\mathrm{abc}}$ & 0.018 \\
\hline LA TotEV (ml) & $28.80 \pm 4.63$ & $30.11 \pm 3.84$ & $35.03 \pm 4.19^{a b}$ & $31.82 \pm 1.66^{\mathrm{ac}}$ & $<0.001$ \\
\hline LA PassEV (ml) & $19.06 \pm 4.93$ & $18.89 \pm 3.82$ & $16.24 \pm 2.97^{\mathrm{ab}}$ & $18.53 \pm 2.89$ & 0.011 \\
\hline LA ActEV (ml) & $9.74 \pm 4.15$ & $11.21 \pm 4.43$ & $18.79 \pm 4.53^{\mathrm{ab}}$ & $13.29 \pm 3.88^{a c}$ & $<0.001$ \\
\hline
\end{tabular}

LAVmax: left atrium maximum volume; LAVImax: left atrium maximum volume index; LAVmin: left atrium minimum volume; LAVImin: left atrium minimum volume index; LAVpre: left atrium pre-contraction volume; LAVIpre: left atrium pre-contraction volume index; LA TotEV: left atrium total emptying volume; LA PassEV: left atrium passive emptying volume; LA ActEV: left atrium active emptying volume

${ }^{\mathrm{a}} p<0.05$ for normal geometry; ${ }^{\mathrm{b}} p<0.05$ for concentric remodeling; ${ }^{\mathrm{c}} p<0.05$ for concentric hypertrophy

and 3). Patients with concentric hypertrophy had higher 3DE-LAVmin and 3DE-LAVImin than those with normal geometry, concentric remodeling, and eccentric hypertrophy, as well as higher 2DE- LAVmin and 2DE-LAVImin than those with normal geometry and concentric remodeling $(P<0.05)$ (Figs. 2 and 3$)$. Additionally, 2DE-and 3DE-LAVpre and LAVIpre were significantly different in patients with left ventricular geometry $(P<0.05)$ (Figs. 2 and 3$)$.

Notably, 2DE-LA TotEV was greater in patients with concentric hypertrophy than in those with normal geometry and concentric remodeling; 3DE-LA TotEV was greater in patients with concentric hypertrophy than in those with normal geometry, concentric remodeling, and 


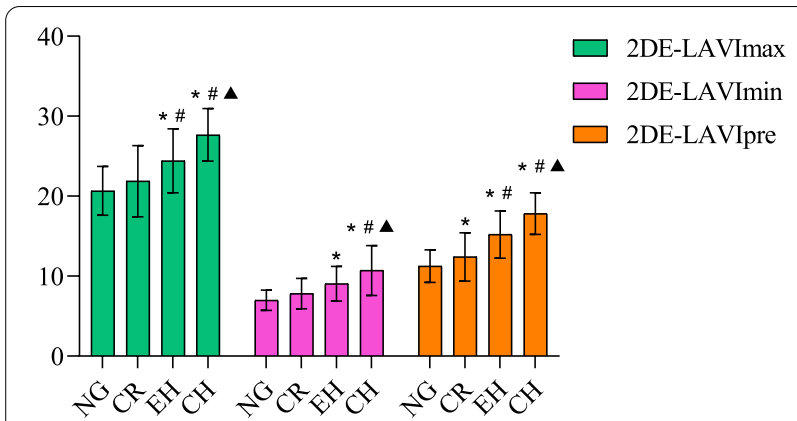

Fig. 2 The comparison of 2DE-left atrium volume indices (LAVImax, LAVImin, LAVIpre) among the different left ventricular geometric patterns. NG: Normal geometry; CR: Concentric remodeling; EH: Eccentric hypertrophy; $\mathrm{CH}$ : Concentric hypertrophy; ${ }^{*}<<0.05$ versus NG; ${ }^{P} P<0.05$ versus $\mathrm{CR} ; \boldsymbol{\Delta} P<0.05$ versus $\mathrm{EH}$

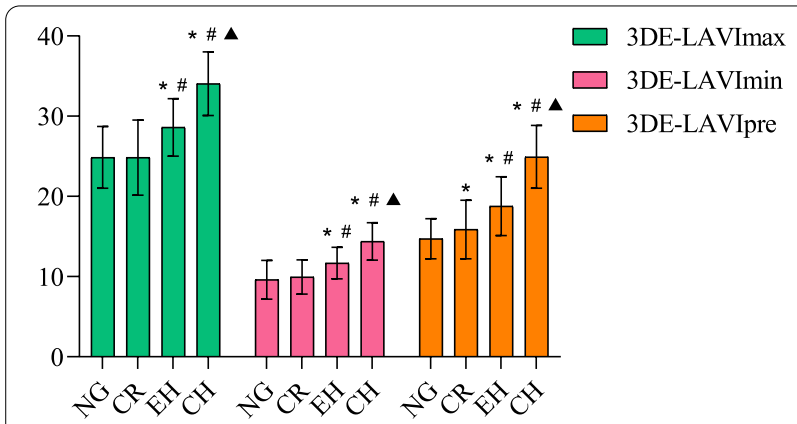

Fig. 3 The comparison of 3DE-left atrium volume indices (LAVImax, LAVImin, LAVIpre) among the different left ventricular geometric patterns. NG: Normal geometry; CR: Concetric remodeling; EH: Eccentric hypertrophy; $\mathrm{CH}$ : Concentric hypertrophy; ${ }^{*} P<0.05$ versus NG; ${ }^{P} P<0.05$ versus $\mathrm{CR}$; $\boldsymbol{\Delta} P<0.05$ versus $\mathrm{EH}$

eccentric hypertrophy; 2DE- and 3DE-LA TotEV were greater in eccentric hypertrophy than in normal geometry. The 3DE-LA PassEV was lower in patients with concentric hypertrophy than in those with normal geometry and concentric remodeling. However, 3DE-LA PassEV showed no differences among the left ventricular geometric patterns. Patients with concentric hypertrophy and eccentric hypertrophy demonstrated a significantly larger 2DE-LA ActEV compared to those with normal geometry and concentric remodeling. Furthermore, patients with concentric hypertrophy revealed a significantly larger 3DE-LA ActEV compared to any of the left ventricular geometric patterns, and larger eccentric hypertrophy compared to those with normal geometry.

The trend of change in left ventricular strains was similar to the change observed in LA volumes. The LAS-S and LAS-E values progressively decreased from normal geometry to concentric hypertrophy; however, LAS-A gradually increased. Patients with concentric hypertrophy and eccentric hypertrophy had higher
LAS-S and LAS-E than those with normal geometry and concentric remodeling. The LAS-A was higher among patients with concentric hypertrophy than in those presenting any left ventricular geometric pattern $(P<0.05)$ (Fig. 4).

\section{Left atrium phasic function in the four left ventricular geometric patterns}

As shown in Table 4, the LA reservoir, estimated by LA total emptying fraction (LA TotEF) and conduit function, estimated by LA passive emptying fraction (LA PassEF), gradually decreased from normal geometry to concentric hypertrophy. Nevertheless, LA booster function, estimated by LA active emptying fraction (LA ActEF), gradually increased in a similar direction. Patients with concentric hypertrophy had lower 2DELA TotEF compared to those with normal geometry and lower 3DE-LA TotEF compared to those with normal geometry and concentric remodeling (Figs. 5 and 6). Patients with concentric hypertrophy and eccentric hypertrophy had lower 2DE-LA PassEF compared to those with normal geometry and concentric remodeling. In addition, patients with concentric hypertrophy had lower 3DE-LA PassEF compared to those with normal geometry and concentric remodeling, and lower 3DE-LA PassEF in patients with eccentric hypertrophy compared to those with normal geometry (Figs. 5 and 6). Patients with concentric hypertrophy had higher 3DE-LA ActEF values than those with normal geometry. However, 2DE-LA ActEF showed no difference among the left ventricular geometric patterns (Figs. 5 and 6).

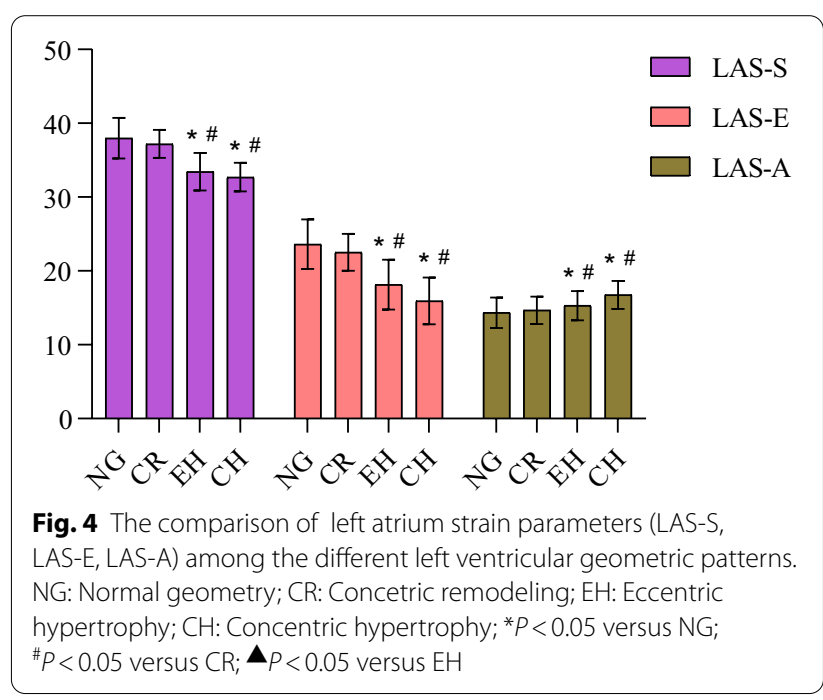


Table 4 Left atrium phasic function in the four left ventricular geometric patterns

\begin{tabular}{|c|c|c|c|c|c|}
\hline & $N G(n=110)$ & $C R(n=56)$ & $\mathrm{CH}(\mathrm{n}=32)$ & $\mathrm{EH}(n=23)$ & $P$ \\
\hline \multicolumn{6}{|l|}{ Reservoir function } \\
\hline 2DE-LA TotEF (\%) & $0.66 \pm 0.04$ & $0.64 \pm 0.04$ & $0.62 \pm 0.09^{a}$ & $0.63 \pm 0.04$ & $<0.001$ \\
\hline 3DE-LA TotEF (\%) & $0.61 \pm 0.07$ & $0.61 \pm 0.04$ & $0.58 \pm 0.05^{\mathrm{ab}}$ & $0.59 \pm 0.03$ & 0.009 \\
\hline 2DE-LAS-S (\%) & $37.97 \pm 2.74$ & $37.20 \pm 1.89$ & $32.70 \pm 1.93^{\mathrm{ab}}$ & $33.44 \pm 2.53^{\mathrm{ab}}$ & $<0.001$ \\
\hline \multicolumn{6}{|l|}{ Conduit function } \\
\hline 2DE-LA PassEF (\%) & $0.46 \pm 0.05$ & $0.44 \pm 0.05$ & $0.36 \pm 0.04^{\mathrm{ab}}$ & $0.38 \pm 0.04^{\mathrm{ab}}$ & $<0.001$ \\
\hline 3DE-LA PassEF (\%) & $0.40 \pm 0.08$ & $0.39 \pm 0.08$ & $0.31 \pm 0.06^{\mathrm{ab}}$ & $0.35 \pm 0.07^{\mathrm{a}}$ & $<0.001$ \\
\hline 2DE-LAS-E (\%) & $23.62 \pm 3.36$ & $22.52 \pm 2.48$ & $15.95 \pm 3.16^{\mathrm{ab}}$ & $18.13 \pm 3.37^{\mathrm{ab}}$ & $<0.001$ \\
\hline \multicolumn{6}{|l|}{ Pump function } \\
\hline 2DE-LA ActEF (\%) & $0.37 \pm 0.08$ & $0.37 \pm 0.08$ & $0.41 \pm 0.13$ & $0.40 \pm 0.05$ & 0.098 \\
\hline 3DE-LA ActEF (\%) & $0.35 \pm 0.13$ & $0.36 \pm 0.10$ & $0.42 \pm 0.06^{\mathrm{a}}$ & $0.37 \pm 0.06$ & 0.011 \\
\hline 2DE-LAS-A (\%) & $14.35 \pm 2.06$ & $14.68 \pm 1.85$ & $16.75 \pm 1.89^{\mathrm{ab}}$ & $15.31 \pm 1.98^{c}$ & $<0.001$ \\
\hline
\end{tabular}

LA TotEF: left atrium total emptying fraction; LA PassEF: left atrium passive emptying fraction; LA ActEF: left atrium active emptying fraction; LAS-S: left atrium strain during systole; LAS-E: left atrium strain during early diastole; LAS-A: left atrium strain during late diastole

${ }^{\mathrm{a}} p<0.05$ for normal geometry; ${ }^{\mathrm{b}} p<0.05$ for concentric remodeling; ${ }^{c} p<0.05$ for concentric hypertrophy

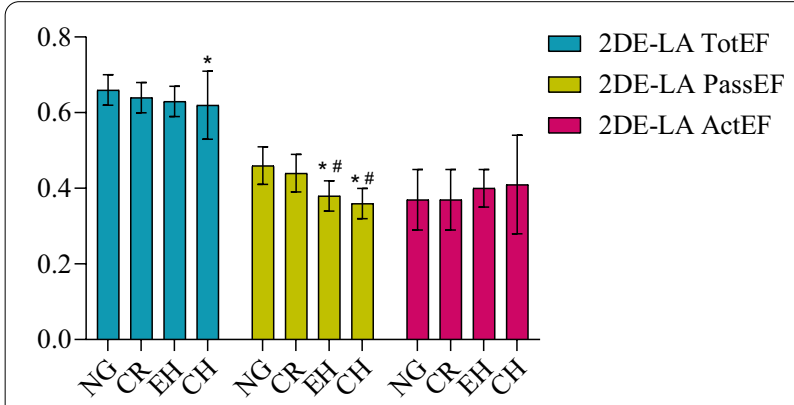

Fig. 5 The comparison of 2DE-left atrium emptying fraction (TotEF, PassEF, ActEF) among the different left ventricular geometric patterns. NG: Normal geometry; CR: Concetric remodeling; EH: Eccentric hypertrophy; $\mathrm{CH}$ : Concentric hypertrophy; ${ }^{*} P<0.05$ versus NG; ${ }^{\#} P<0.05$ versus $C R$

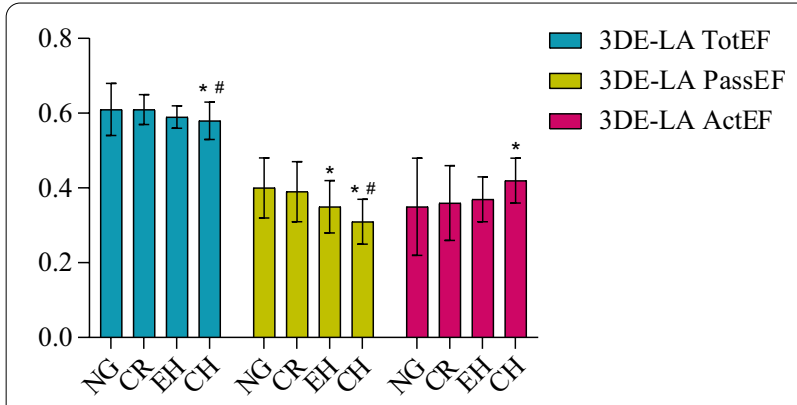

Fig. 6 The comparison of 3DE- left atrium emptying fraction (TotEF, PassEF, ActEF) among the different left ventricular geometric patterns. NG: Normal geometry; CR: Concetric remodeling; EH: Eccentric hypertrophy; $\mathrm{CH}$ : Concentric hypertrophy; ${ }^{*} P<0.05$ versus NG; ${ }^{\#} P<0.05$ versus $C R$

\section{Multiple linear regression analysis of the relationship between the left ventricular geometry and LAVImax and LA phasic function}

In order to clarify the independent influence of the left ventricular geometric patterns on LAVImax and LA phasic function, we performed multiple linear regression analyses, in which age, gender, BMI, SBP, diabetes mellitus, dyslipidemia, AHI, and LVMI were included in the model. As shown in Table 5, concentric hypertrophy was significantly associated with 2DE-LAVI$\max (\beta=0.449,95 \% \mathrm{CI}: 3.592-7.436 ; P<0.001)$, while concentric and eccentric hypertrophy were associated with 3DE-LAVImax $(\beta=0.539, \quad 95 \%$ CI: $5.946-9.761$; $P<0.001$ and $\beta=0.137,95 \% \mathrm{CI}: 0.290-4.306 ; P=0.025$, respectively). Subsequently, concentric hypertrophy was associated with 2DE- and 3DE-LA TotEF $(\beta=-0.219$, 95\%CI: $-0.074-(-0.001) ; \quad P=0.046$ and $\beta=-0.276$, 95\%CI: $-0.073-(-0.010) ; P=0.010$, respectively). Moreover, concentric hypertrophy and eccentric hypertrophy were associated with 2DE-LA PassEF $(\beta=-0.422$, 95\%CI: $-0.148-(-0.052) ; P<0.001$ and $\beta=-0.221,95 \% \mathrm{CI}$ : -0.111- (-0.010); $P=0.019$, respectively), while concentric hypertrophy, eccentric hypertrophy, and concentric remodeling were associated with 3DE-LA PassEF $(\beta=-0.589,95 \%$ CI: $-0.126-(-0.070) ; P<0.001, \beta=-0.382$, 95\%CI: $-0.103-(-0.044) ; P<0.001$, and $\beta=-0.138,95 \%$ CI: -0.034- (-0.003); $P=0.018$, respectively). Only concentric hypertrophy was associated with 3DE-LA ActEF $(\beta=0.239$, 95\%CI: 0.005-0.144; $P=0.035)$. Furthermore, concentric and eccentric hypertrophy were associated with 3DE-LAS-S, 3DE-LAS-E, and 3DE-LAS-A (all, $P<0.05)$. 


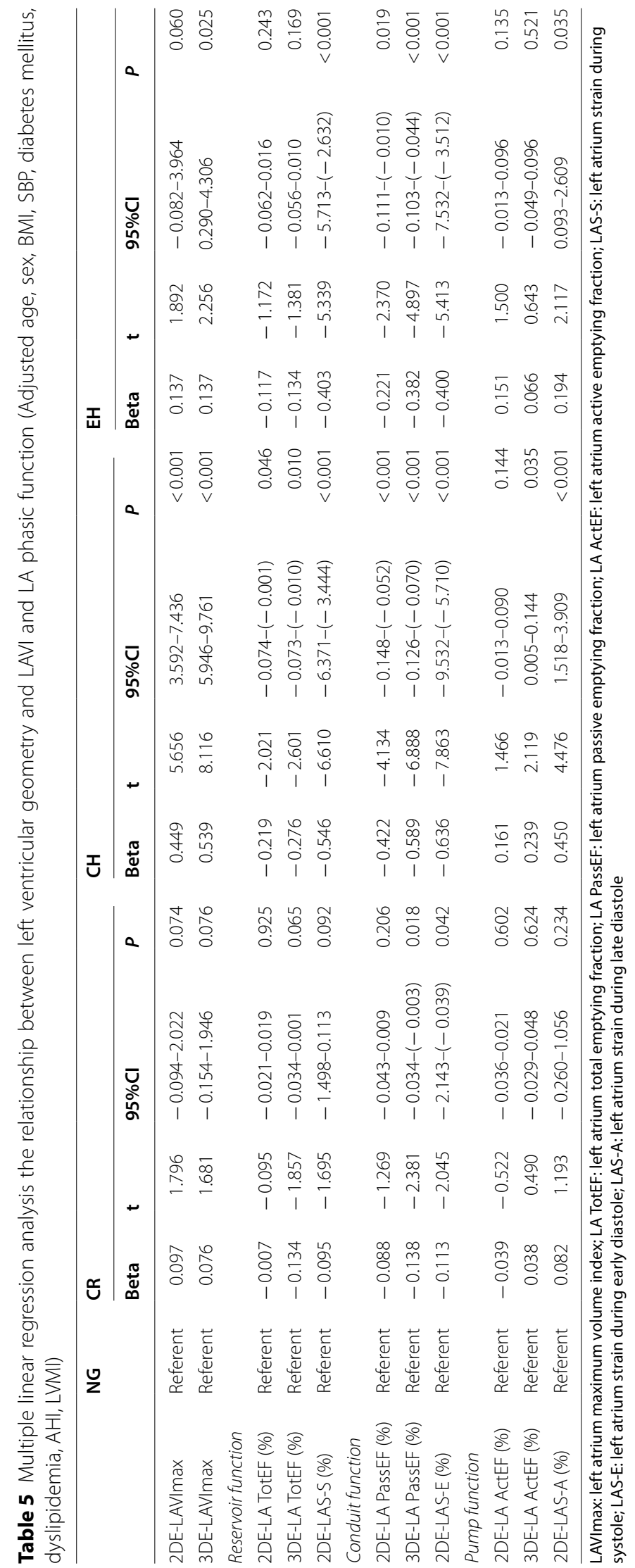




\section{Discussion}

To our knowledge, this is the first study to evaluate the influence of left ventricular geometry on LA volume and phase function in patients with OSAS combined with multiple echocardiographic methods. Our main findings include the following: (1) LA volumes and indices showed an increasing pattern from normal geometry to concentric hypertrophy, and 2DE-LAVImax was associated with concentric hypertrophy, while 3DE-LAVImax was associated with concentric hypertrophy and eccentric hypertrophy; (2) LA reservoir and conduit function gradually increased from normal geometry to concentric hypertrophy, while LA booster pump function gradually decreased in a similar direction; (3) multiple linear regression analysis revealed that concentric hypertrophy was significantly associated with LA phasic function, and $3 \mathrm{DE}$ parameters were significantly associated with left ventricular geometric patterns compared to $2 \mathrm{DE}$ parameters.

An enlarged LA was associated with increased severity of OSAS and was evaluated by LA volume using $2 \mathrm{DE}$ and 3DE. Nevertheless, changes in LA volume remain unclear in patients with OSAS based on left ventricular geometry. Our findings showed that LA volumes and indices gradually increased from normal geometry to concentric hypertrophy. This may have been due to left ventricular overload and impaired myocardial relaxation caused by nocturnal apnea-hypopnea events, which may have triggered left ventricular diastolic dysfunction $[20,21]$. Moreover, left ventricular diastolic function gradually decreased from normal geometry to concentric hypertrophy, resulting in increased left ventricular filling pressure and blocked LA blood flow into the left ventricle, and may explain the change in LA volume. Importantly, LAVImax played a vital role in predicting cardiovascular adverse events and was included in the guidelines [16]. However, previous findings on the relationship between LAVImax and left ventricular geometry are inconsistent. The results varied in that LAVImax was only related to eccentric hypertrophy, concentric hypertrophy, or both, and not related to left ventricular geometry [7-10]. The varying results among different studies may be due to differences in duration and degree of hypertension and the state of treatment.

Furthermore, the present study demonstrated that only concentric hypertrophy was associated with 2DE-LAVImax. This study was the same as the previous study, in which individual left ventricular geometric patterns were related to LAVImax; meanwhile, differences in our study may be due to differences in type of disease, blood pressure, and age. Importantly, we evaluated LAVImax using $3 \mathrm{DE}$, suggesting that concentric and eccentric hypertrophy were associated with 3DE-LAVImax. This was due to the fact that 3DE was more precise than $2 \mathrm{DE}$ in the measurement of left ventricular volume and highly valuable in predicting the incidence of cardiovascular adverse events [16]. Our results confirm that the incidence of cardiovascular events and clinical prognosis differ in concentric and eccentric hypertrophy [22].

Previous studies evaluated the phasic function of LA using 3DE and 2D-STE, respectively. Consequently, they demonstrated that OSAS induces LA remodeling and dysfunction [23, 24]. Nevertheless, our study further revealed that LA phasic function was based on left ventricular geometry.

In addition, we found that LA reservoir function, estimated by LA TotEF and LAS-S, gradually decreased from normal geometry to concentric hypertrophy. This result may have been due to the fact that the hypertrophic wall of the left ventricle increased compression of the coronary artery and oxygen consumption of hypertrophic cardiomyocytes, causing hypoxia damage and sensitivity to ischemia of cardiomyocytes, further inducing fibrosis and decreased compliance in the left atrium [25]. Notably, this phenomenon is apparent with a larger LVMI. Furthermore, LVMI increased from a normal geometry to concentric hypertrophy. In contrast, LA TotEF decreased, and the LAS-S showed similar results. Multiple linear regression analysis revealed that concentric hypertrophy was significantly associated with LA reservoir function.

This investigation also demonstrated that LA conduit function, evaluated by LA PassEF and LAS-E, gradually decreased from normal geometry to concentric hypertrophy. Despite not being statistically significant, Oliveira et al. previously showed that 3DE-LA PassEF decreased in patients with OSAS compared to the control group [23]. Altekin et al. assessed LA phasic function using 2D-STE and reported that LAS-E gradually decreased from control to severe OSAS and was associated with AHI [24]. Similarly, Tadic and colleagues revealed similar findings that the LA conduit function decreased in patients with hypertension [26]. Here, SBP, DBP, and AHI gradually increased from normal geometry to concentric hypertrophy. Our results are consistent with those of previous studies. Multiple linear regression showed that concentric and eccentric hypertrophy were associated with 2DE- and 3DE-LA PassEF, and LA strain confirmed these results.

The LA booster pump function, evaluated by LA ActEF and LAS-A, gradually increased from normal geometry to concentric hypertrophy. Previous studies have shown that OSAS causes an increase in LA booster pump function [23, 24]. In addition, using velocity vector imaging, Yang et al. found that patients with hypertension and left ventricular hypertrophy had 
reduced LA conduit function and enhanced booster pump function [27]. Our results are in line with those of a previous study, possibly due to the compensatory increase in LA booster pump function, which is essential to maintain normal left ventricular diastolic filling with a decrease in LA reservoir and conduit function. Multiple linear regression revealed that concentric hypertrophy was associated with 3DE-LA ActEF; meanwhile, it was not associated with 2DE-LA ActEF. The LA strain validated the 3DE results, showing that concentric hypertrophy was associated with LAS-A. This was primarily due to the fact that the LA strain was more useful for the prediction of adverse cardiovascular events than the 2DE LA volume and more sensitive to these events. Furthermore, this could explain why patients with left ventricular hypertrophy and higher LVMI have increased LA booster pump function than those without left ventricular hypertrophy.

Our study has the following limitations. Due to its cross-sectional nature, we could not ascertain a direct causal relationship between left ventricular geometry and LA phase function; hence, further longitudinal studies are necessary. Also, the study could be carried out using the new classifications proposed by Dallas et al. [28] due to the relatively small sample size. Changes in LA phase function need to be further investigated in the future using a new classification system.

\section{Conclusions}

Our findings suggest that LA phasic function changes with left ventricular geometry. Moreover, the reservoir and conduit function of the LA gradually decreased from normal geometry to concentric hypertrophy; nevertheless, the function of the booster pump gradually increased. Furthermore, concentric hypertrophy had the most significant negative impact on LAVImax and LA phasic function.

\footnotetext{
Abbreviations

OSAS: Obstructive sleep apnea syndrome; AHI: Apnea-hypopnea index; $\mathrm{SaO}_{2}$ : Oxygen saturation; 2DE: Two-dimensional echocardiography; 3DE: Three-dimensional echocardiography; 2D-STE: Two-dimensional speckletracking echocardiography; LAVImax: Left ventricular maximum volume index; LAVImin: Left ventricular minimum volume index; LAVIpre: Left ventricular precontraction volume index; LA TotEF: Left ventricular total emptying fraction; LA PassEF: Left ventricular passive emptying fraction; LA ActEF: Left ventricular active emptying fraction; LV: Left ventricle.
}

\section{Supplementary Information}

The online version contains supplementary material available at https://doi. org/10.1186/s12872-021-02018-1.

Additional file 1. Ethical approval.

Additional file 2. Certificate of english editing.

\section{Acknowledgements}

We would like to thank Feng Xiaoli, Cui Tong, and Kang Caihong for their assistance with this study and English editing company of editage for language (Additional file 2).

\section{Authors' contributions}

$J W$ and $Y Z$ participated in the study. $Y Z$ performed the statistical analyses and drafted the manuscript. ZZ performed the PSG technology. WS, JL, and YT collected the data. All authors read and approved the final manuscript.

\section{Funding}

This work was supported by the Fund from Shanxi 1331 Project Key Innovative Research Team (1331KIRT) and the Shanxi Provincial Natural Science Foundation of China (201901D111361).

\section{Availability of data and materials}

The datasets analyzed during the current study are available from the corresponding author upon request.

\section{Declarations}

Ethics approval and consent to participate

This study was approved by the Institutional Ethics Committee of the First Hospital of Shanxi Medical University (No. K-ZKO24) and performed in accordance with the Declaration of Helsinki and its later amendments. Please view the ethical approval in Additional file 1. Written informed consent was obtained from all patients included in this study.

Consent for publication

Not applicable.

\section{Competing interests}

The authors declare that they have no conflicts of interest.

\section{Author details}

${ }^{1}$ Medical imaging department of Shanxi Medical University; Department of Ultrasound, First Hospital of Shanxi Medical University, 85 Jiefang Nan Road, Taiyuan 030001, Shanxi, China. ${ }^{2}$ Department of Respiratory, First Hospital of Shanxi Medical University, 85 Jiefang Nan Road, Taiyuan 030001, Shanxi, China.

Received: 6 December 2020 Accepted: 13 April 2021

Published online: 24 April 2021

\section{References}

1. Lévy P, Kohler M, McNicholas W, Barbé F, McEvoy R, Somers V, Lavie L, Pépin J. Obstructive sleep apnoea syndrome. Nat Rev Dis Primers. 2015;1:15015. 
2. Cuspidi C, Tadic M, Sala C, Gherbesi E, Grassi G, Mancia G. Targeting concentric left ventricular hypertrophy in obstructive sleep apnea syndrome. A meta-analysis of echocardiographic studies. Am J f Hypertens. 2020;33(4):310-5.

3. Bodez D, Damy T, Soulat-Dufour L, Meuleman C, Cohen A. Consequences of obstructive sleep apnoea syndrome on left ventricular geometry and diastolic function. Arch Cardiovasc Dis. 2016;109:494-503.

4. Holtstrand Hiälm H, Fu M, Hansson PO, Zhong Y, Caidahl K, Mandalenakis Z, Morales D, Ergatoudes C, Rosengren A, Grote L, Thunström E. Association between left atrial enlargement and obstructive sleep apnea in a general population of 71-year-old men. J Sleep Res. 2017;27(2):254-60.

5. Lavie P, Lavie L. Cardiovascular morbidity and mortality in obstructive sleep apnea. Curr Pharm Des. 2008;14(32):3466-73.

6. Lavie CJ, Patel DA, Milani RV, Ventura HO, Shah S, Gilliland Y. Impact of echocardiographic left ventricular geometry on clinical prognosis. Prog Cardiovasc Dis. 2014;57(1):3-9.

7. Gerdts E, Oikarinen L, Palmieri V, Otterstad JE, Wachtell K, Boman K, Dahlof $B$, Devereux RB. Correlates of left atrial size in hypertensive patients with left ventricular hypertrophy: the Losartan Intervention For Endpoint Reduction in Hypertension (LIFE) Study. Hypertension. 2002;39(3):739.

8. Cioffi G, Mureddu GF, Stefenelli C, Simone GD. Relationship between left ventricular geometry and left atrial size and function in patients with systemic hypertension. J Hypertens. 2004;22(8):1589-96.

9. Patel DA, Lavie CJ, Milani RV, Gilliland Y, Shah S, Ventura HO. Association of left ventricular geometry with left atrial enlargement in patients with preserved ejection fraction. Congest Heart Fail. 2012;18(1):4-8.

10. Tsioufis C, Taxiarchou E, Syrseloudis D, Chatzis D, Stefanadis C. Left ventricular mass but not geometry determines left atrial size in the early stages of hypertension. J Hum Hypertens. 2009;23(10):674-9.

11. Thomas AL, Muraru FD, Bap E, Sitges FM, Rosca EM, Gp H, Myh I, Donal $J E, L p b c D$. Evaluation of left atrial size and function: relevance for clinical practice-ScienceDirect. J Am Soc Echocardiogr. 2020;33(8):934-52.

12. Berry R, Budhiraja R, Gottlieb D, Gozal D, Iber C, Kapur V, Marcus C, Mehra R, Parthasarathy S, Quan S, et al. Rules for scoring respiratory events in sleep: update of the 2007 AASM Manual for the Scoring of Sleep and Associated Events. Deliberations of the Sleep Apnea Definitions Task Force of the American Academy of Sleep Medicine. J Clin Sleep Med. 2012;8(5):597-619.

13. Chobanian A, Bakris G, Black H, Cushman W, Green L, Izzo J, Jones D, Materson B, Oparil S, Wright J, et al. Seventh report of the Joint National Committee on Prevention, Detection, Evaluation, and Treatment of High Blood Pressure. Hypertension (Dallas, Tex: 1979). 2003;42(6):1206-52.

14. Xia Y, Fu Y, Wang Y, Qian Y, Li X, Xu H, Zou J, Guan J, Yi H, Meng L, et al. Prevalence and predictors of atherogenic serum lipoprotein dyslipidemia in women with obstructive sleep apnea. Sci Rep. 2017;7:41687.

15. Kapur VK, Auckley DH, Chowdhuri S, Kuhlmann DC, Mehra R, Ramar K, Harrod CG. Clinical practice guideline for diagnostic testing for adult obstructive sleep apnea: an american academy of sleep medicine clinical practice guideline. J Clin Sleep Med. 2017;13(03):479-504.

16. Lang RM, Badano LP, Mor-Avi V, Afilalo J, Armstrong A, Ernande L, Flachskampf FA, Foster E, Goldstein SA, Kuznetsova T, Lancellotti P. Recommendations for cardiac chamber quantification by echocardiography in adults: an update from the American Society of Echocardiography and the European Association of Cardiovascular Imaging (vol 28, pg 1, 2015). J Am Soc Echocardiogr. 2016;29(3):276-276.

17. Badano LP, Kolias TJ, Muraru D, Abraham TP, Aurigemma G, Edvardsen T, D'Hooge J, Donal E, Fraser AG, Marwick T. Standardization of left atrial, right ventricular, and right atrial deformation imaging using two-dimensional speckle tracking echocardiography: a consensus document of the EACVI/ASE/Industry Task Force to standardize deformation imaging. Eur Heart J Cardiovasc Imaging. 2018;19(6):591-600.

18. Anwar AM, Soliman O, Geleijnse ML, Nemes A, Vletter WB, Cate F. Assessment of left atrial volume and function by real-time three-dimensional echocardiography. Int J Cardiol. 2008;123(2):155-61.

19. Blume GG, Mcleod CJ, Barnes ME, Seward JB, Pellikka PA, Bastiansen PM, Tsang TSM. Left atrial function: physiology, assessment, and clinical implications. Eur J Echocardiogr. 2011;12(6):421-30.

20. Somers VK, Dyken ME, Clary MP, Abboud FM. Sympathetic neural mechanisms in obstructive sleep apnea. J Clin Investig. 1995;96(4):1897-904.

21. Thomas JJ, Ren J. Obstructive sleep apnoea and cardiovascular complications: perception versus knowledge. Clin Exp Pharmacol Physiol. 2012;39(12):995-1003.

22. Patel DA, Lavie CJ, Milani RV, Ventura HO. Left atrial volume index predictive of mortality independent of left ventricular geometry in a large clinical cohort with preserved ejection fraction. Mayo Clin Proc Mayo Clin. 2011;86(8):730-7.

23. Oliveira W, Campos O, Lira-Filho EB, Cintra FD, Vieira M, Ponchirolli A, Paola AD, Tufik S, Poyares D. Left atrial volume and function in patients with obstructive sleep apnea assessed by real-time three-dimensional echocardiography. J Am Soc Echocardiogr. 2008;21(12):1355-61.

24. Altekin RE, Yanikoglu A, Karakas MS, Ozel D, Kucuk M, Yilmaz H, Demir I. Assessment of left atrial dysfunction in obstructive sleep apnea patients with the two dimensional speckle-tracking echocardiography. Clin Res Cardiol. 2012:101(6):403-13.

25. Chen L, Zhang J, Gan TX, Chen-Izu Y, Hasday JD, Karmazyn M, Balke CW, Scharf SM. Left ventricular dysfunction and associated cellular injury in rats exposed to chronic intermittent hypoxia. J Appl Physiol. 2008;104(1):218-23.

26. Tadic M, Cuspidi C, Pencic B, Kocijancic V, Celic V. The influence of left ventricular geometry on left atrial phasic function in hypertensive patients. Blood Press. 2015;24(6):1-8.

27. Yang L, Qiu Q, Fang SH. Evaluation of left atrial function in hypertensive patients with and without left ventricular hypertrophy using velocity vector imaging. Int J Cardiovasc Imaging. 2014;30(8):1465-71.

28. Khouri MG, Peshock RM, Ayers CR, Lemos JD, Drazner MH. A 4-tiered classification of left ventricular hypertrophy based on left ventricular geometry: the Dallas Heart Study. Circ Cardiovasc Imaging. 2010;3(2):164-71.

\section{Publisher's Note}

Springer Nature remains neutral with regard to jurisdictional claims in published maps and institutional affiliations.
Ready to submit your research? Choose BMC and benefit from:

- fast, convenient online submission

- thorough peer review by experienced researchers in your field

- rapid publication on acceptance

- support for research data, including large and complex data types

- gold Open Access which fosters wider collaboration and increased citations

- maximum visibility for your research: over 100M website views per year

At BMC, research is always in progress.

Learn more biomedcentral.com/submissions 